\title{
BCG vaccination history associates with decreased SARS-CoV-2 seroprevalence across a diverse cohort of health care workers
}

\author{
Magali Noval Rivas, ${ }^{1,2}$ Joseph E. Ebinger, ${ }^{3,4}$ Min Wu, ${ }^{3,4}$ Nancy Sun, ${ }^{3,4}$ Jonathan Braun, ${ }^{5}$ Kimia Sobhani, ${ }^{6}$ Jennifer E. Van Eyk, ${ }^{3,7,8}$ \\ Susan Cheng, ${ }^{3,4,7}$ and Moshe Arditi $1,2,4$ \\ 'Department of Pediatrics, Division of Infectious Diseases and Immunology, Infectious and Immunologic Diseases Research Center (IIDRC), and Department of Biomedical Sciences, Cedars-Sinai Medical \\ Center, Los Angeles, California, USA. ${ }^{2}$ Department of Pediatrics, David Geffen School of Medicine at UCLA, Los Angeles, California, USA. ${ }^{3}$ Department of Cardiology, ${ }^{4}$ Smidt Heart Institute, ${ }^{5} \mathrm{~F}$. Widjaja \\ Foundation Inflammatory Bowel and Immunobiology Research Institute, ${ }^{6}$ Pathology and Laboratory Medicine, ${ }^{7}$ Advanced Clinical Biosystems Institute, and ${ }^{8}$ Barbra Streisand Women's Heart Center, Cedars- \\ Sinai Medical Center, Los Angeles, California, USA.
}

BACKGROUND. Severe acute respiratory syndrome coronavirus 2 (SARS-CoV-2) has caused more than 1 million deaths worldwide; thus, there is an urgent need to develop preventive and therapeutic strategies. The antituberculosis vaccine bacillus Calmette-Guérin (BCG) demonstrates nonspecific, protective innate immune-boosting effects. Here, we determined whether a history of BCG vaccination was associated with decreased SARS-CoV-2 infection and seroconversion in a longitudinal, retrospective observational study of a diverse cohort of health care workers (HCWs).

\begin{abstract}
METHODS. We assessed SARS-CoV-2 seroprevalence and collected medical questionnaires, which included information on BCC vaccination status and preexisting demographic and clinical characteristics, from an observational cohort of HCWs in a multisite Los Angeles health care organization. We used multivariate analysis to determine whether a history of BCC vaccination was associated with decreased rates of SARS-CoV-2 infection and seroconversion.
\end{abstract}

RESULTS. Of the $6201 \mathrm{HCWs,} 29.6 \%$ reported a history of BCG vaccination, whereas $68.9 \%$ had not received BCG vaccination. Seroprevalence of anti-SARS-CoV-2 IgC as well as the incidence of self-reported clinical symptoms associated with coronavirus disease 2019 (COVID-19) were markedly decreased among HCWs with a history of BCC vaccination compared with those without BCG vaccination. After adjusting for age and sex, we found that a history of BCG vaccination, but not meningococcal, pneumococcal, or influenza vaccination, was associated with decreased SARS-CoV-2 IgC seroconversion.

CONCLUSIONS. A history of BCG vaccination was associated with a decrease in the seroprevalence of anti-SARS-CoV-2 IgG and a lower number of participants who self-reported experiencing COVID-19-related clinical symptoms in this cohort of HCWs. Therefore, large randomized, prospective clinical trials of BCC vaccination are urgently needed to confirm whether BCG vaccination can confer a protective effect against SARS-CoV-2 infection.

\section{Introduction}

As of early October 2020, more than 37.5 million people worldwide had been infected by the novel severe acute respiratory syndrome coronavirus 2 (SARS-CoV-2), which has resulted in more than 1 million deaths due to coronavirus disease 2019 (COVID-19) (1). SARS-CoV-2 infection may present as an asymptomatic or mild disease, and the majority of infected individuals develop typical COVID-19 clinical features such as high fever, dry cough, chills,

\section{Delated Commentary: https://doi.org/10.1172/JCl145545}

Authorship note: SC and MA contributed equally to this work.

Conflict of interest: The authors have declared that no conflict of interest exists.

Copyright: (5) 2021, American Society for Clinical Investigation.

Submitted: October 14, 2020; Accepted: November 18, 2020; Published: January 19, 2021

Reference information: J Clin Invest. 2021;131(2):e145157.

https://doi.org/10.1172/JCl145157. reduced sense of smell or taste, muscle aches, shortness of breath, and fatigue (2). However, $15 \%-20 \%$ of infected patients progress to severe or critical COVID-19 and develop serious lung damage and breathing difficulties, which may lead to respiratory failure due to secondary bacterial or fungal infections, and death (2-4). SARSCoV-2 was first discovered in Wuhan, China, more than 8 months ago $(3,5)$, and, to date, no curative or protective treatments have been identified. Despite public health measures implemented to minimize the spread of the virus, the global population continues to experience high levels of exposure to SARS-CoV-2.

Intense efforts are underway to accelerate the development of safe and effective COVID-19 vaccines that can be used for mass immunization. However, given the time these clinical trials require, it has been proposed that existing live, attenuated viral vaccines could be leveraged to provide nonspecific immune protection as a bridge to mitigate the SARS-CoV-2 pandemic until targeted vaccines become available. 


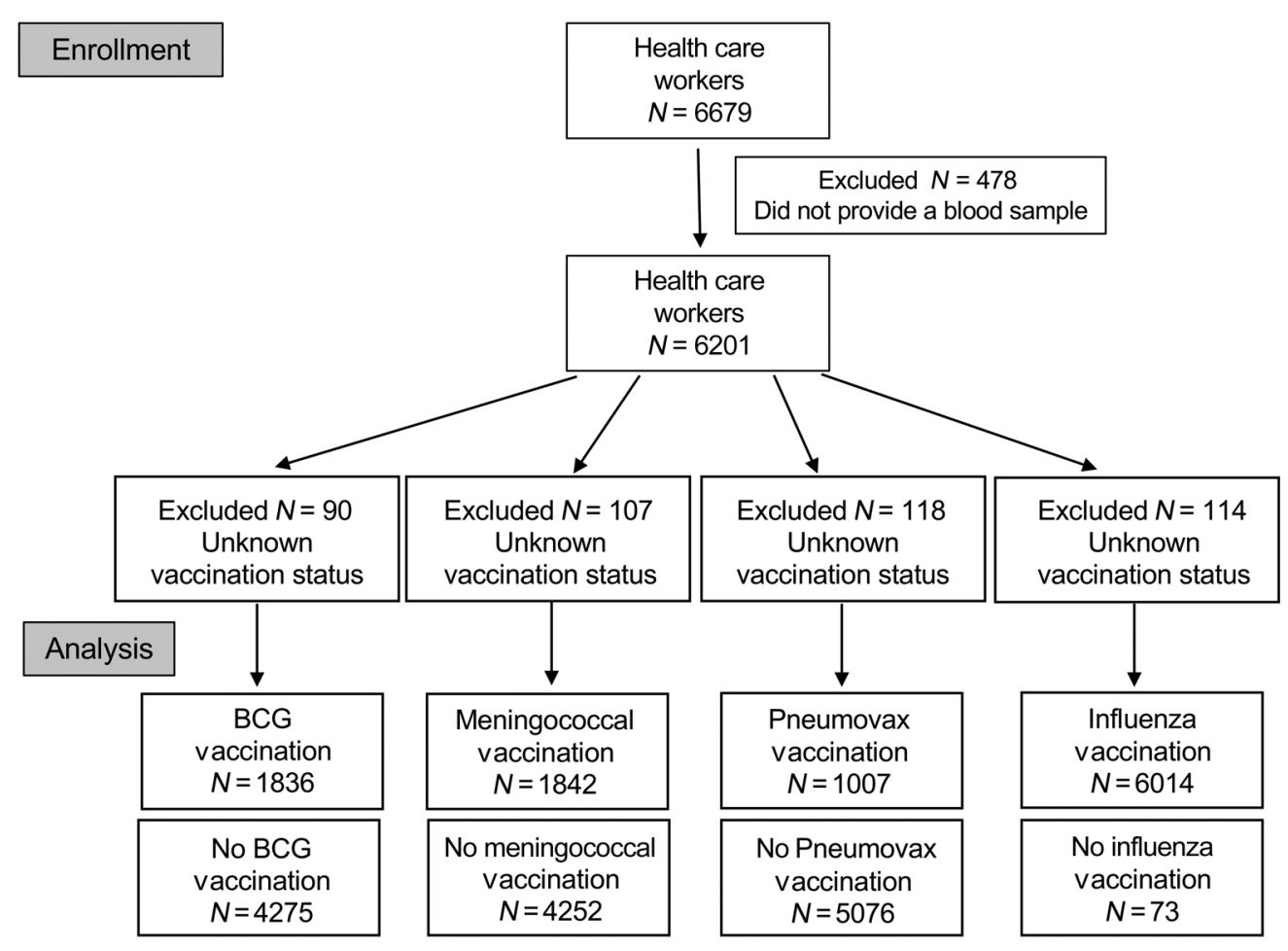

Figure 1. Flow chart of this retrospective observational study. A total of $6679 \mathrm{HCWs}$ were recruited in this study. Self-reported information about a previous COVID-19 diagnosis, a positive or negative SARS-CoV-2 RT-PCR test, experience of COVID-19-related symptoms, as well as BCC, meningococcal, Pneumovax, and influenza vaccination status were collected retrospectively with an electronic survey. Among the 6679 participants, 478 did not provide a blood sample to measure the anti-SARS-CoV-2 IgG index and were thus excluded from the analysis. Of the remaining 6201 participants, 1836 reported a previous history of BCG vaccination, 1842 reported a previous meningococcal vaccination, 1007 reported receiving a Pneumovax vaccination, and 6014 reported receiving an influenza vaccination.

Bacillus Calmette-Guérin (BCG) is a live, attenuated vaccine strain of Mycobacterium bovis used against tuberculosis; however, various studies have demonstrated that the BCG vaccine also induces potent and nonspecific protection against other unrelated diseases (6). Early administration of the BCG vaccine reduces child mortality by approximately $38 \%-45 \%$, mainly as a result of decreased neonatal sepsis, respiratory infections, and fever (712). Nonspecific effects of BCG vaccination are not limited to children, as BCG vaccination in adolescents leads to a $70 \%$ decrease in the incidence of respiratory tract infections (13), and BCG-vaccinated elderly individuals (60-75 years of age) also experience fewer respiratory infections (14). BCG vaccination confers protection in multiple animal models experimentally challenged with Herpesvirus hominis (15), Staphylococcus aureus, Yersinia pestis, Klebsiella pneumonia, or Schistosoma mansoni (reviewed in ref. 16). The beneficial heterologous effects of BCG vaccination against unrelated viral respiratory infections are mediated by enhancement of the innate immune response and induction of innate immune memory, a process also known as trained immunity (17). Trained immunity is regulated by transcriptional, epigenetic, and metabolic reprogramming of innate immune cells such as monocytes, macrophages, and NK cells, which results in increased transcription of genes involved in pathogen recognition and antimicrobial inflammatory responses. After BCG vaccination, the epigenetic-mediated nonspecific immune benefits last at least 1 year (17), and protection against tuberculosis has been shown to last at least 20 years (18). Therefore, the demonstrated ability of the BCG vaccine - as well as other live, attenuated vaccines including the live oral polio vaccine (OPV) (19) and the measles mumps rubella (MMR) vaccine (20) - to activate nonspecific innate immune responses may provide a unique opportunity to reset the course of the current pandemic.

Given the evidence that the BCG vaccine is protective not only against tuberculosis but also unrelated viral infections, we hypothesized that BCG vaccination might be beneficial by reducing both the susceptibility to and severity of SARS-CoV-2 infection in particularly vulnerable groups such as health care workers (HCWs). As part of a rapid scientific response to the present public health crisis, on March 18, 2020, we convened a collaborative of frontline clinicians and scientists to form the Coronavirus Risk Associations and Longitudinal Evaluation (CORALE) studies (coralestudy.org). We established 2 base study cohorts with enrollment centered on (a) patients with suspected or confirmed COVID-19 treated in our health system (currently $n>8300$ ) and (b) HCWs directly or indirectly involved in delivering their care (currently $n=$ 6201). Here, we retrospectively analyzed data collected from this large cohort of HCWs to determine whether a history of BCG vaccination was associated with altered seroprevalence of and infection with SARS-CoV-2 (21).

Our results demonstrate that anti-SARS-CoV-2 IgG seroprevalence and the number of participants who self-reported experiencing COVID-19 symptoms were lower among HCWs with a his- 


\section{Table 1. Demographics and clinical characteristics of the HCWs cohort} based on BCG vaccination status

\begin{tabular}{|c|c|c|c|c|}
\hline & \multicolumn{4}{|c|}{ Number (\%) } \\
\hline & $\begin{array}{c}\text { Overall } \\
(n=6201)\end{array}$ & $\begin{array}{c}\text { BCG vaccinated } \\
(n=1836)\end{array}$ & $\begin{array}{l}\text { Not BCG vaccinated } \\
\qquad(n=4275)\end{array}$ & $P$ value \\
\hline Age (yr), mean (SD) & $41.46(12.01)$ & $43.15(12.03)$ & $40.64(11.86)$ & $<0.001$ \\
\hline Sex & & & & 0.013 \\
\hline Male & 1989 (32.1) & $546(29.7)$ & $1411(33.0)$ & \\
\hline Female & $4212(67.9)$ & $1290(70.3)$ & $2864(67.0)$ & \\
\hline Self-reported ethnicity & & & & $<0.001$ \\
\hline Hispanic & 1187 (19.1) & $264(14.4)$ & 895 (20.9) & \\
\hline Non-Hispanic & $4721(76.1)$ & $1490(81.2)$ & $3173(74.2)$ & \\
\hline Other & $293(4.7)$ & $82(4.5)$ & $207(4.8)$ & \\
\hline Self-reported race & & & & $<0.001$ \\
\hline American Indian or Alaska Native & $17(0.3)$ & $4(0.2)$ & $13(0.3)$ & \\
\hline Asian & $1912(30.8)$ & $849(46.2)$ & $1031(24.1)$ & \\
\hline Black or African American & $379(6.1)$ & $108(5.9)$ & $263(6.2)$ & \\
\hline Native Hawaiian or Pacific Islander & $53(0.9)$ & $19(1.0)$ & $31(0.7)$ & \\
\hline White & $3111(50.2)$ & $642(35.0)$ & 2431 (56.9) & \\
\hline Other & $729(11.8)$ & $214(11.7)$ & $506(11.8)$ & \\
\hline \multicolumn{5}{|l|}{ COVID-19-related symptoms } \\
\hline Fever & $871(14.4)$ & $234(13.0)$ & $633(15.0)$ & 0.047 \\
\hline Chills & $1108(18.3)$ & 306 (17.0) & 797 (18.9) & 0.09 \\
\hline Dry cough & $1732(28.7)$ & $477(26.5)$ & $1249(29.7)$ & 0.012 \\
\hline Shortness of breath & $843(14.0)$ & $224(12.5)$ & $617(14.7)$ & 0.024 \\
\hline Muscle aches & $1460(24.2)$ & $386(21.5)$ & $1066(25.3)$ & 0.002 \\
\hline Fatigue & $1932(32.1)$ & $498(27.8)$ & $1428(33.9)$ & $<0.001$ \\
\hline Reduced sense of smell or taste & $458(7.6)$ & $100(5.6)$ & $357(8.5)$ & $<0.001$ \\
\hline No symptoms/signs of COVID-19 & $4502(73.6)$ & $1385(75.6)$ & 3099 (72.7) & 0.017 \\
\hline Self-reported COVID-19 diagnosis & $157(2.5)$ & $34(1.9)$ & $122(2.9)$ & 0.029 \\
\hline \multicolumn{5}{|l|}{ Positive SARS-CoV-2 test } \\
\hline Self-reported RT-PCR & $93(1.5)$ & $19(1.0)$ & $73(1.7)$ & 0.062 \\
\hline Anti-SARS-CoV-2 IgG & $217(3.5)$ & $50(2.7)$ & $162(3.8)$ & 0.044 \\
\hline Anti-SARS-CoV-2 IgG index & & & & 0.019 \\
\hline IgC index $\leq 0.4$ & 5904 (95.2) & $1767(96.2)$ & $4053(94.8)$ & \\
\hline IgG index $>0.4$ & $297(4.8)$ & $69(3.8)$ & $222(5.2)$ & \\
\hline
\end{tabular}

tory of BCG vaccination. These findings support the pursuit of the 22 registered clinical trials (ClinicalTrials.gov) already in progress to explore the therapeutic and preventive potential of prospective BCG vaccination for SARS-CoV-2 infection and COVID-19 severity (BCG Vaccine for Health Care Workers as Defense Against COVID-19 [BADAS] study, NCT04348370; Efficacy of BCG Vaccination in the Prevention of COVID-19 via the Strengthening of Innate Immunity in Health Care Workers [COVID-BCG], NTC04384549; and BCG Vaccination to Protect Healthcare Workers Against COVID-19 [BRACE], NCT04327206).

\section{Results}

Study population. To determine whether a history of BCG vaccination might be beneficial in preventing SARS-CoV-2 infection and COVID-19 severity, we retrospectively analyzed a cohort of HCWs who were recruited in the Cedars-Sinai Health System network in the Los Angeles County area (21). Among the 6679 HCWs who participated in this study, a total of 6201 individuals provided a blood sample for SARS-CoV-2 serology testing and answered an electronic survey regarding past medical history and status of BCG, influenza, meningococcal, and pneumococcal (Pneumovax) vaccination (Figure 1). Our cohort consisted of a majority of women $(67.9 \%$ women; $32.1 \%$ men; Table 1).

COVID-19 was declared a pandemic by the WHO on March 11, 2020. By early March 2020, the Los Angeles County Public Health Department had already documented 145 cases of COVID-19, and as of mid-September 2020, more than 243,400 COVID-19 cases had been reported (22). Our study and sample collection spanned from May 11, 2020 to June 18, 2020, with $3.5 \%$ of the individuals in our cohort testing positive for SARS-CoV-2 IgG antibodies (Table 1 and Figure 2A). In addition to considering an IgG index cutoff point of 1.4 or higher as seropositive, as recommended by the test manufacturer, we also conducted analyses using the cutoff point of 0.4 or higher to define seropositive status within this more sensitive range, given other reports (23) and our own laboratory findings of this threshold being strongly associated with prior COVID-19 diagnosis and symptoms. Notably, our main results were consistent for analyses using either the more stringent 1.4 or higher threshold or the more sensitive 0.4 or higher threshold. Using the more sensitive 0.4 or higher threshold, the overall seroprevalence of anti-SARS-CoV- 2 antibodies in our cohort was $4.8 \%$ (Table 1). Of the 6201 individuals in our cohort, 1836 reported a history of BCG vaccination, 4275 had never received BCG vaccination, and the $B C G$ vaccination status was unknown in 90 individuals (Table 1 and Figure 2B). The BCG-vaccinated group was older (43.1 \pm 12.03 years) than those in the non-BCG-vaccinated group ( $40.6 \pm 11.86$ years) (Figure $2 \mathrm{C}$ ).

History of $B C G$ vaccination is associated with fewer self-reports of COVID-19-related symptoms. To determine whether a history of BCG vaccination is associated with a protective beneficial effect and decreases the severity of COVID-19-related symptoms, we analyzed the responses provided by each participant through an electronic survey. Compared with individuals who were not BCG vaccinated, those with a history of BCG vaccination were less likely to report experiencing COVID-19-related symptoms over the previous 6 months $(75.6 \%$ vs. $72.7 \% ; P=0.017$; Figure 2D). We focused on fever, chills, dry cough, shortness of breath, muscle aches, fatigue, and reduced of sense of smell or taste as COVID-19-related symptoms, based on emerging data and CDC guidelines $(24,25)$. The most commonly reported COVID-19related symptoms were fatigue, dry cough, and muscle aches (Table 1 and Figure 2E). Remarkably, participants with a history of BCG vaccination were less likely than non-BCG-vaccinated participants to report any of those symptoms in the 6 months prior to their enrollment in the study (Table 1 and Figure 2E). 
A Positive anti-SARS-CoV-2 lgG test

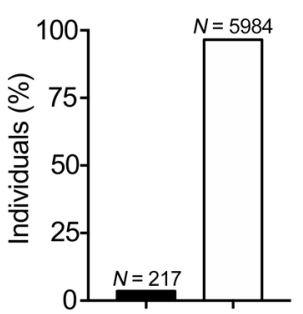

Positive SARS-CoV-2 lgG Negative SARS-CoV-2 IgG

D No symptoms/signs of COVID-19

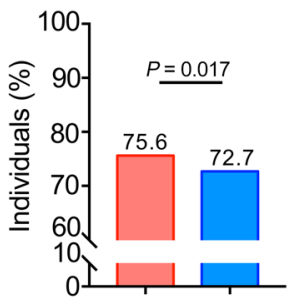

$\square$ BCG vaccinated $\square$ Not BCG vaccinated

G Self-reported positive SARS-CoV-2

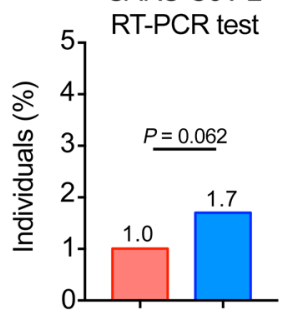

B

Vaccination status

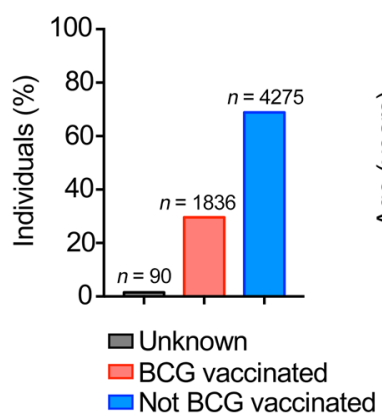

C

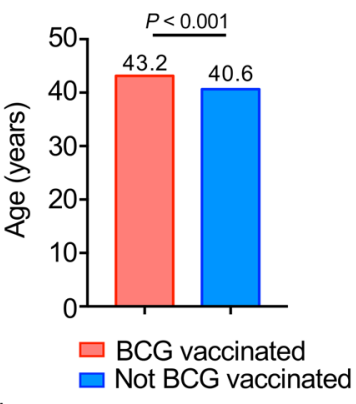

$\mathbf{E}$

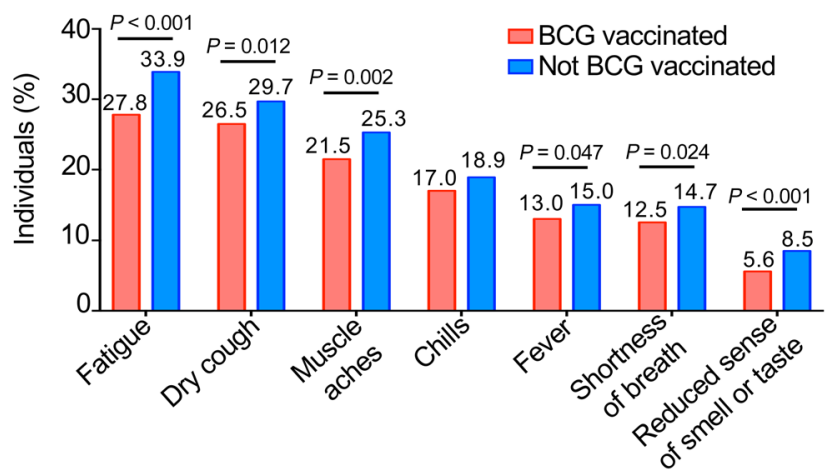

F Self-reported COVID-19 diagnosis

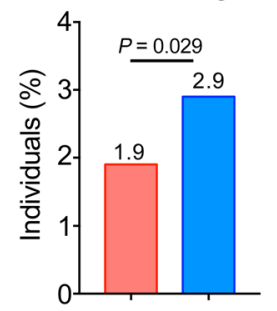

$\square$ BCG vaccinated $\square$ Not BCG vaccinated
H

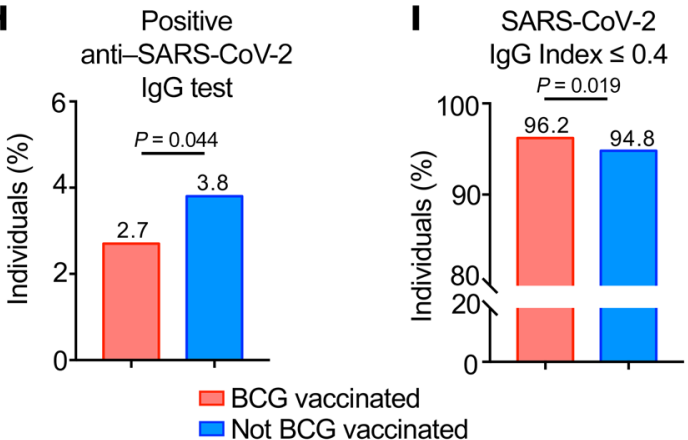

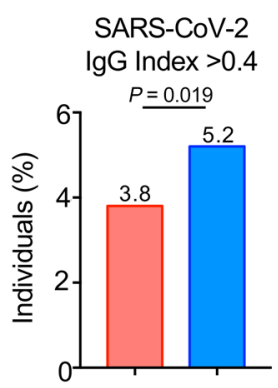

SARS-CoV-2

$\lg G$ Index $>0.4$

Figure 2. History of BCG vaccination is associated with fewer reports of COVID-19-related symptoms and decreased SARS-CoV-2 infection. (A) Percentage of participants in the cohort of HCWs $(n=6201)$ with a positive anti-SARS-CoV-2 IgC test. (B) Percentage of individuals in the whole cohort who reported a history of BCG vaccination $(n=1836)$ or absence of a BCG vaccination $(n=4275)$. (C) Age (mean) of the participants reporting a previous history of BCG vaccination or no BCG vaccination. (D) Percentage of participants with or without a history of BCG vaccination who reported an absence of COVID-19-related symptoms. (E) Percentage of individuals with or without a history of BCG vaccination who self-reported experiencing COVID-19-related symptoms during the preceding 6 months. (F) Percentage of participants with or without a history of BCG vaccination who self-reported a previous COVID-19 diagnosis from a MD or RT-PCR test. (G) Percentage of participants with or without a history of BCG vaccination who self-reported having a positive SARS-CoV-2 RT-PCR test. (H) Percentage of participants with or without a history of BCG vaccination with a positive anti-SARS-CoV-2 IgC test result. (I) Percentage of individuals with or without a history of BCG vaccination with a SARS-CoV-2 IgC index threshold of 0.4 or lower or higher than 0.4 . $P<0.05$, by $t$ test $(\mathbf{C})$ or $\chi^{2}$ test $(\mathbf{D}-\mathbf{I}) ; P$ values are displayed in each graph.

History of BCG vaccination is associated with decreased SARSCoV-2 infections and seroprevalence. We next assessed whether a history of BCG vaccination was associated with a decreased incidence of SARS-CoV-2 infection by analyzing the proportion of individuals in our cohort who self-reported either a positive COVID-19 diagnosis by a medical doctor (MD) or a positive SARSCoV-2 reverse transcription PCR (RT-PCR) test. The percentage of individuals with a COVID-19 diagnosis or a positive SARSCoV-2 RT-PCR test was markedly reduced for those who reported a history of BCG vaccination compared with those who did not receive BCG vaccination (Table 1 and Figure 2, F and G). Similarly, the percentage of individuals with a serology test positive for SARS-CoV-2 (IgG) was significantly lower for BCG-vaccinated individuals than those who were not BCG vaccinated (at all levels of the IgG index) (Table 1 and Figure 2, $\mathrm{H}$ and I).

Participants with certain underlying preexisting comorbidities such as hypertension, diabetes, cardiovascular disease, or chronic obstructive pulmonary disease (COPD) are at increased risk of 


\section{Table 2. Self-reported preexisting comorbidities reported by HCWs based on BCG vaccination status}

\begin{tabular}{lccc} 
Comorbidities & $\begin{array}{c}\text { BCG vaccinated } \\
(\boldsymbol{n}=\mathbf{1 8 3 6 )}\end{array}$ & $\begin{array}{c}\text { Number (\%) } \\
\text { Not BCG vaccinated } \\
(\boldsymbol{n}=\mathbf{4 2 7 5 )}\end{array}$ & $\boldsymbol{P}$ value \\
Hypertension & $334(18.8)$ & $678(16.2)$ & 0.016 \\
Cardiovascular disease & $50(2.9)$ & $81(2.0)$ & 0.043 \\
Diabetes mellitus & $165(9.3)$ & $218(5.3)$ & $<0.001$ \\
COPD & $36(2.0)$ & $49(1.2)$ & 0.016 \\
\hline
\end{tabular}

developing severe COVID-19 $(26,27)$. In our cohort of HCWs, we found that those with a history of BCG vaccination had significantly more comorbidities such as hypertension, cardiovascular disease, diabetes, and COPD than did those without a history of BCG vaccination (Table 2). Remarkably, despite reporting an increased frequency of comorbidities, the group with a history of BCG vaccination had less seroconversion and a significantly lower anti-SARS-CoV-2 IgG index (Figure 2I). Overall, our data indicate that a history of BCG vaccination is associated with decreased rates of SARS-COV-2 infection and seropositivity, indicating that BCG vaccination might be protective against SARS-CoV-2 infection.

A decreased occurrence of COVID-19-related symptoms and SARS-CoV-2 infection is associated only with a history of $B C G$ vaccination. The medical survey answered by each participant also contained questions regarding the status of 3 other common vaccines: meningococcal, Pneumovax, and influenza. We therefore asked if a history of those vaccinations was also associated with a beneficial protective effect against COVID-19 symptoms and/or SARS-CoV-2 infection. Among the 6201 participants who provided a blood sample and answered the medical survey, 1842 reported having received the meningococcal vaccine, 1007 had received the Pneumovax vaccine, and 6014 reported having received an influenza vaccine (Tables 3,4 , and 5 , and Figure 3A). The majority of HCWs participating in this study reported being vaccinated for influenza (96.9\%) and were more likely to experience some of the COVID-19-related symptoms than the non-influenza-vaccinated subjects (Table 5 and Figure 3B). However, the very low frequency $(<1.2 \%)$ of HCWs who were not vaccinated for influenza may have limited statistical power for comparisons made with influenza-vaccinated individuals, and the results of these analyses suggested no substantial between-groups differences. In contrast to our findings for subjects with a history of BCG vaccination (Figure 2E), the frequencies of other COVID-19-related symptoms did not consistently differ on the basis of vaccination status for the meningococcal or pneumococcal agents (Tables 3-5 and Figure 3B). The percentage of individuals who experienced COVID-19 symptoms or self-reported a COVID-19-positive diagnosis - either by a MD or a positive RT-PCR test - did not differ in terms of pneumococcal or influenza vaccination status, indicating that those vaccines might not provide a beneficial protective effect against SARS-CoV-2 infection (Tables 4 and 5, and Figure 3, B-E). Notably, a history of meningococcal vaccination was significantly associated with an increase in self-reported COVID-19 medical diagnoses (Table 3 and Figure 3D). The percentage of individuals who self-reported a positive SARS-CoV-2 RT-PCR test, as well as the percentage of individuals with an antiSARS-CoV-2 IgG index of 0.4 or lower or an index higher than 0.4, did not differ with regard to the vaccination status of any of those 3 vaccines (Tables 3-5 and Figure 3, F and G).

We next constructed separate multivariable models, each adjusted for age and sex, to analyze the associations of BCG, meningococcal, Pneumovax, and influenza vaccination history with a SARS-CoV-2 IgG index of 0.4 or higher (Table 6). Only a history of BCG vaccination was significantly associated with lower SARS-CoV-2 IgG index values (Table 6). The protective effect of prior BCG vaccination was persistent when the risks of SARS-CoV-2 exposure were added to age- and sex-controlled models $(\mathrm{OR}=0.74, \mathrm{CI}, 0.56-0.98 ; P=0.039)$. SARS-CoV-2 exposure risk was assessed on the basis of the location at which

Table 3. Demographic and clinical characteristics of the HCW cohort based on meningococcal vaccination status

\begin{tabular}{|c|c|c|c|}
\hline & \multicolumn{3}{|c|}{ Number (\%) } \\
\hline & $\begin{array}{c}\text { Meningococcal } \\
\text { vaccinated }(n=1842)\end{array}$ & $\begin{array}{c}\text { Not meningococcal } \\
\text { vaccinated }(n=4252)\end{array}$ & $P$ value \\
\hline Age (yr), mean (SD) & $36.01(9.53)$ & $43.73(12.17)$ & $<0.001$ \\
\hline Sex & & & 0.72 \\
\hline Male & $598(32.5)$ & 1359 (32.0) & \\
\hline Female & $1244(67.5)$ & $2893(68.0)$ & \\
\hline Self-reported ethnicity & & & $<0.001$ \\
\hline Hispanic & $300(16.3)$ & $856(20.1)$ & \\
\hline Non-Hispanic & $1436(78.0)$ & $3213(75.6)$ & \\
\hline Other & $106(5.8)$ & $183(4.3)$ & \\
\hline Self-reported race & & & 0.163 \\
\hline American Indian or Alaska Native & $2(0.1)$ & $14(0.3)$ & \\
\hline Asian & $598(32.5)$ & $1280(30.1)$ & \\
\hline Black or African American & $107(5.8)$ & $260(6.1)$ & \\
\hline Native Hawaiian or Pacific Islander & $10(0.5)$ & $40(0.9)$ & \\
\hline White & $908(49.3)$ & $2155(50.7)$ & \\
\hline Other & $217(11.8)$ & $503(11.8)$ & \\
\hline \multicolumn{4}{|l|}{ COVID-19-related symptoms } \\
\hline Fever & $281(15.4)$ & $586(14.0)$ & 0.16 \\
\hline Chills & $367(20.1)$ & $733(17.5)$ & 0.019 \\
\hline Dry cough & $518(28.5)$ & $1208(29.0)$ & 0.72 \\
\hline Shortness of breath & $273(15.0)$ & $566(13.6)$ & 0.15 \\
\hline Muscle aches & $443(24.3)$ & $1005(24.1)$ & 0.89 \\
\hline Fatigue & $635(34.9)$ & $1287(30.9)$ & 0.002 \\
\hline Reduced sense of smell or taste & $145(8.0)$ & $310(7.5)$ & 0.52 \\
\hline No symptoms/signs of COVID-19 & $1333(72.5)$ & $3139(74.0)$ & 0.24 \\
\hline Self-reported COVID-19 diagnosis & $60(3.3)$ & $96(2.3)$ & 0.029 \\
\hline \multicolumn{4}{|l|}{ Positive SARS-CoV-2 test } \\
\hline Self-reported RT-PCR & $34(1.8)$ & $58(1.4)$ & 0.19 \\
\hline Anti-SARS-CoV-2 IgC & $72(3.9)$ & $140(3.3)$ & 0.26 \\
\hline Anti-SARS-CoV-2 lgC index & & & 0.64 \\
\hline $\lg G$ index $\leq 0.4$ & $1750(95.0)$ & 4050 (93.5) & \\
\hline IgG index $>0.4$ & $92(5.0)$ & $199(4.7)$ & \\
\hline
\end{tabular}




\section{Table 4. Demographics and clinical characteristics of the HCWs cohort used in this study based on Pneumovax vaccination status}

\begin{tabular}{|c|c|c|c|}
\hline & \multicolumn{3}{|c|}{ Number (\%) } \\
\hline & $\begin{array}{c}\text { Pneumovax } \\
\text { vaccinated }(n=1007)\end{array}$ & $\begin{array}{c}\text { Not Pneumovax } \\
\text { vaccinated }(n=5076)\end{array}$ & $P$ value \\
\hline Age (yr), mean (SD) & $45.64(14.77)$ & 40.58 (11.16) & $<0.001$ \\
\hline Sex & & & $<0.001$ \\
\hline Male & $383(38.0)$ & $1572(31.0)$ & \\
\hline Female & $624(62.0)$ & $3504(69.0)$ & \\
\hline Self-reported ethnicity & & & 0.02 \\
\hline Hispanic & $151(15.0)$ & $1003(19.8)$ & \\
\hline Non-Hispanic & $811(80.5)$ & $3832(75.5)$ & \\
\hline Other & $45(4.5)$ & $241(4.7)$ & \\
\hline Self-reported race & & & 0.008 \\
\hline American Indian or Alaska Native & $4(0.4)$ & $13(0.3)$ & \\
\hline Asian & $350(34.8)$ & $1522(30.0)$ & \\
\hline Black or African American & $68(6.8)$ & $302(5.9)$ & \\
\hline Native Hawaiian or Pacific Islander & $7(0.7)$ & $42(0.8)$ & \\
\hline White & $485(48.2)$ & $2575(50.7)$ & \\
\hline Other & $93(9.2)$ & $622(12.3)$ & \\
\hline \multicolumn{4}{|l|}{ COVID-19-related symptoms } \\
\hline Fever & $148(15.0)$ & $713(14.2)$ & 0.58 \\
\hline Chills & $180(18.3)$ & $915(18.3)$ & 1.00 \\
\hline Dry cough & $278(28.1)$ & $1438(28.8)$ & 0.71 \\
\hline Shortness of breath & $155(15.8)$ & $682(13.7)$ & 0.09 \\
\hline Muscle aches & $238(24.1)$ & $1208(24.2)$ & 0.96 \\
\hline Fatigue & $328(33.2)$ & $1586(31.8)$ & 0.42 \\
\hline Reduced sense of smell or taste & $73(7.4)$ & $382(7.7)$ & 0.85 \\
\hline No symptoms/signs of COVID-19 & $738(73.5)$ & $3723(73.5)$ & 1.00 \\
\hline Self-reported COVID-19 diagnosis & $25(2.5)$ & $132(2.6)$ & 0.92 \\
\hline \multicolumn{4}{|l|}{ Positive SARS-CoV-2 test } \\
\hline Self-reported RT-PCR & $18(1.8)$ & $75(1.5)$ & 0.55 \\
\hline Anti-SARS-CoV-2 IgC & $35(3.5)$ & $179(3.5)$ & 1.00 \\
\hline Anti-SARS-CoV-2 IgG index & & & 0.65 \\
\hline $\lg G$ index $\leq 0.4$ & $962(95.5)$ & $4829(95.1)$ & \\
\hline IgG index $>0.4$ & $45(4.5)$ & $247(4.9)$ & \\
\hline
\end{tabular}

comorbidities, such as hypertension, diabetes, or serious heart diseases, are at increased risk of developing severe COVID-19 (26-28), indicating that a defective innate antiviral immune response may contribute to SARS-CoV-2 susceptibility. There are currently no curative therapeutics or preventive treatments for COVID-19, and the number of COVID-19-related deaths continues to rise worldwide.

BCG vaccination was initially developed to protect against tuberculosis, but recent epidemiological studies have demonstrated that it also significantly reduces infant mortality (12), apparently owing to nonspecific effects of BCG against other respiratory tract viral infections and neonatal sepsis (9-12). The BCG vaccine's nonspecific and long-term heterologous protection against other viral pathogens is partly mediated by trained immunity through epigenetic, transcriptional, and functional reprograming of myeloid cells (17). In a double-blind, randomized trial of either BCG or placebo vaccination in elderly patients, BCG vaccination was correlated with a delayed and decreased incidence of new infections, particularly respiratory tract infections (29). Since BCG vaccination-mediated trained immunity boosts antiviral immune responses, it has been suggested that BCG-induced trained immunity might be an efficient preventive measure against SARS-CoV-2 infection and COVID-19 severity (30-32). Indeed, several epidemiological studies have suggested that countries with a national policy of BCG vaccination have a reduced prevalence of COVID-19 incidence and mortality $(33,34)$. However, we are still lacking clinical data to confirm this observation and definitively demonstrate a BCG-mediated protective effect against COVID-19.

Here, in a large cohort of HCWs employed in a multisite major health care system network of Los Angeles County (21), we found that a history of BCG vaccination was associated with fewer reports of COVID-19-related symptoms as well as a reduced frequency of SARS-CoV-2 infection, as demonstrated by

the HCWs worked, such as a dedicated COVID-19 intensive care unit (ICU), a non-COVID-19 ICU, a COVID-19-dedicated ward, a non-COVID-19 ward, the emergency department, an outpatient clinic, an administrative office, or a home-based office.

Taken together, these results indicate that a history of BCG vaccination confers a nonspecific protective effect against SARSCoV-2 infection and is associated with fewer self-reports of COVID-19 symptoms. This appears to be specific to BCG, as a history of other vaccinations, such as the meningococcal, Pneumovax, or influenza vaccines, was not associated with a similar protection against SARS-CoV-2 infection.

\section{Discussion}

The majority of SARS-CoV-2-infected individuals develop a mild course of COVID-19 and never require hospitalization, but a small proportion progresses to a more severe form of disease that may lead to acute respiratory distress syndrome and death (3). The elderly and individuals with underlying medical fewer self-reported SARS-CoV-2 RT-PCR-positive tests and/or a lower anti-SARS-CoV-2 IgG titers index. Despite reporting more preexisting comorbidities such as hypertension, diabetes, cardiovascular disease, and COPD, which are known risk factors for the development of severe COVID-19 and associated with a poor prognosis $(26,27)$, individuals with a history of BCG vaccination still had a lower anti-SARS-CoV-2 IgG index, indicative of a protective effect against SARS-CoV-2 infection. Importantly, this protective association appeared to be specific to a history of BCG vaccination, as we did not observe similar results with the 3 other types of vaccines (meningococcal, Pneumovax, and influenza).

In our cohort of HCWs, we detected an absolute $3.5 \%$ rate of anti-SARS-CoV-2 IgG seroprevalence, indicating that a substantial proportion of these participants were previously infected with SARS-CoV-2. This seroprevalence is lower than that reported among HCWs in the New York City area (13.7\%) (35), which experienced a more severe outbreak; however, it is relatively close to the 


\section{Table 5. Demographics and clinical characteristics of the HCWs cohort used in this study based on influenza vaccination status}

\begin{tabular}{|c|c|c|c|}
\hline & \multicolumn{3}{|c|}{ Number (\%) } \\
\hline & $\begin{array}{c}\text { Influenza } \\
\text { vaccinated }(n=6014)\end{array}$ & $\begin{array}{c}\text { Not influenza } \\
\text { vaccinated }(n=73)\end{array}$ & $P$ value \\
\hline Age (yr), mean (SD) & $41.37(11.93)$ & $41.42(14.67)$ & 0.97 \\
\hline Sex & & & 0.61 \\
\hline Male & 1930 (32.1) & $26(35.6)$ & \\
\hline Female & $4084(67.9)$ & $47(64.4)$ & \\
\hline Self-reported ethnicity & & & 0.001 \\
\hline Hispanic & $1142(19.0)$ & $15(20.5)$ & \\
\hline Non-Hispanic & $4595(76.4)$ & $48(65.8)$ & \\
\hline Other & $277(4.6)$ & $10(13.7)$ & \\
\hline Self-reported race & & & 0.017 \\
\hline American Indian or Alaska Native & $17(0.3)$ & $0(0.0)$ & \\
\hline Asian & $1862(31.0)$ & $14(19.2)$ & \\
\hline Black or African American & $356(5.9)$ & $9(12.3)$ & \\
\hline Native Hawailian or Pacific Islander & $49(0.8)$ & $1(1.4)$ & \\
\hline White & $3032(50.4)$ & $34(46.6)$ & \\
\hline Other & $698(11.6)$ & $15(20.5)$ & \\
\hline \multicolumn{4}{|l|}{ COVID-19-related symptoms } \\
\hline Fever & $862(14.5)$ & $5(7.0)$ & 0.11 \\
\hline Chills & $1093(18.4)$ & $8(11.4)$ & 0.18 \\
\hline Dry cough & 1707 (28.9) & $12(17.1)$ & 0.043 \\
\hline Shortness of breath & $822(13.9)$ & $11(15.5)$ & 0.84 \\
\hline Muscle aches & $1438(24.3)$ & $10(14.1)$ & 0.06 \\
\hline Fatigue & $1898(32.1)$ & $21(30.0)$ & 0.80 \\
\hline Reduced sense of smell or taste & $453(7.7)$ & $3(4.3)$ & 0.40 \\
\hline No symptoms/signs of COVID-19 & $4417(73.6)$ & $51(69.9)$ & 0.55 \\
\hline Self-reported COVID-19 diagnosis & $153(2.5)$ & $2(2.7)$ & 1.00 \\
\hline \multicolumn{4}{|l|}{ Positive SARS-CoV-2 test } \\
\hline Self-reported RT-PCR & $92.5(1.5)$ & $1(1.4)$ & 1.00 \\
\hline Anti-SARS-CoV-2 lgG & $211(3.5)$ & $2(2.7)$ & 0.97 \\
\hline Anti-SARS-CoV-2 IgC index & & & 0.58 \\
\hline $\lg G$ index $\leq 0.4$ & $5724(95.2)$ & $71(97.3)$ & \\
\hline $\lg G$ index $>0.4$ & $290(4.8)$ & $2(2.7)$ & \\
\hline
\end{tabular}

seroprevalence previously reported in adults from the Los Angeles County area in mid-April 2020 (36). Our model (statistically adjusted for sex and age) confirmed that only individuals with a history of BCG vaccination, and not meningococcal, Pneumovax, or influenza vaccination, were less likely to have a SARS-CoV-2 IgG index value above 0.4. This observation was made across a range of different COVID-19 exposure risks, including among participants working in high-risk settings such as COVID-19-dedicated ICUs. Furthermore, individuals with a history of BCG vaccination were less likely to report COVID-19-related symptoms. Thus, BCG-vaccinated individuals may have been less sick and therefore produced fewer anti-SARS-CoV-2 antibodies, or they may have mounted a more efficient cellular immune response against SARS-CoV-2.

Through the epigenetic and metabolic reprograming of innate immune cells, particularly monocytes, macrophages, and NK cells, BCG vaccination induces a long-lasting memory and the capacity to respond more strongly upon restimulation (17). This nonspecific boosting of the innate immune response following BCG vaccination has also been reported for other live, attenuated vaccines, including the OPV (19) and MMR (20) vaccines. Indeed, studies have shown that both OPV and MMR vaccines are associated with a decreased number of hospital admissions for infections, including fewer respiratory tract infections (37-39). Therefore, in addition to BCG vaccination, OPV or MMR vaccination may also be considered to mitigate the SARS-CoV-2 pandemic until safe and effective COVID-19-specific vaccines become available.

A limitation of our study is the lack of information regarding the timeline of $\mathrm{BCG}$ vaccination in our cohort. Long-term follow-up studies of a BCG vaccination trial in American Indian and Alaska Native individuals $(40,41)$ as well as a retrospective analysis of United Kingdom (42) and Norwegian (43) cohorts demonstrate that BCG vaccination efficacy can persist over the long term and protect individuals from tuberculosis more than 20 years after delivery. Experimental studies indicate that the effects of BCG vaccination on trained immunity can last from days to months or even years $(6,44$, 45). However, further and more detailed studies are warranted to characterize how long BCG vaccination might confer protection against SARS-CoV-2.

The immune mechanisms by which BCG-induced trained immunity may decrease the incidence of SARS-CoV-2 infection or the severity of COVID-19 will need to be further characterized. By inducing genome-wide epigenetic changes in monocytes, BCG-induced trained immunity may lead to reduced SARS-CoV-2 viremia and increased IL- $1 \beta$ production (46). BCG vaccination also induces long-term trained immunity in NK cells, resulting in their enhanced in vitro production of cytokines, mainly IL-1 $\beta$, after restimulation with either mycobacteria or an unrelated microbial stimulus (47). Severe SARS-CoV-2 infection is associated with decreased numbers of circulating $\mathrm{NK}$ cells and $\mathrm{CD}^{+}$and $\mathrm{CD} 8^{+} \mathrm{T}$ cells, as well as a dysfunctional cytotoxic response $(48,49)$. Those observations indicate a compromised innate immune response during severe COVID-19 that could potentially be boosted by BCG vaccination and its long-term enhancement of innate immune mechanisms. Multiple reports also indicate the presence of SARS-CoV- 2 cross-reacting $\mathrm{T}$ cells, mostly $\mathrm{CD} 4^{+} \mathrm{T}$ cells, in healthy individuals who were not previously exposed to SARS-CoV-2 (50-53). Those cross-reactive T cells might be memory $\mathrm{T}$ cells induced by previous infections with other coronaviruses that cause the common cold and could potentially influence COVID-19 disease severity (54). BCG vaccination induces the activation of $\mathrm{CD} 4^{+} \mathrm{T}$ cells and their production of IFN- $\gamma(55)$, and recent in silico analysis revealed that BCG and SARS-CoV-2 share a common 9-amino acid sequence, suggesting therefore that BCG vaccination could lead to the generation of $\mathrm{T}$ cells that cross-react with SARS-CoV-2 (56).

In some circumstances, such as in autoimmunity or autoinflammatory responses, the increased capacity of innate immune cells to respond more strongly to a stimulus might be harmful by promoting further tissue damage (17). Indeed, monocytes and macrophages isolated from patients with various autoimmune or autoinflammatory disorders similarly 
A Meningococcal

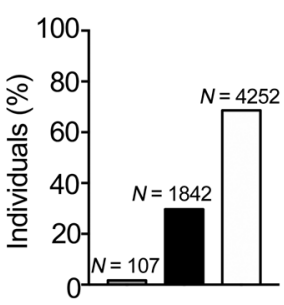

B
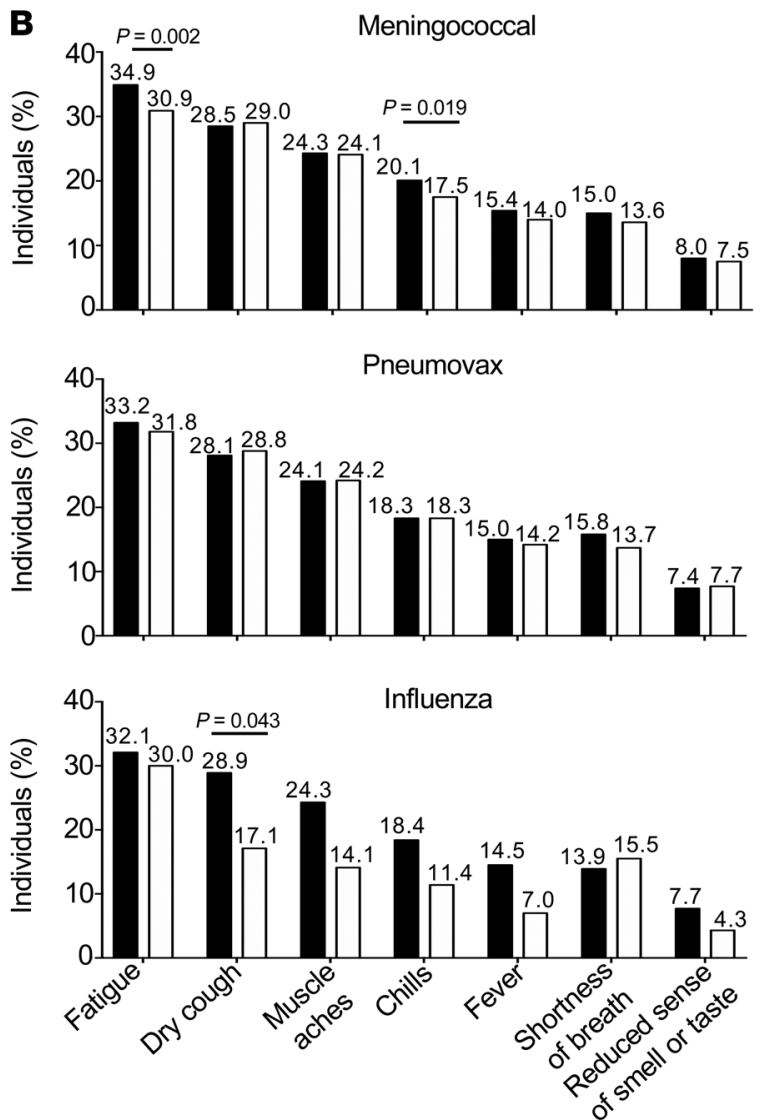

Influenza

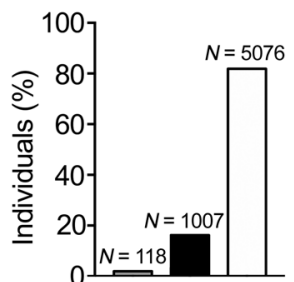

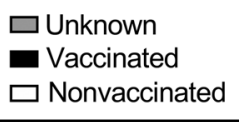

$N=73$

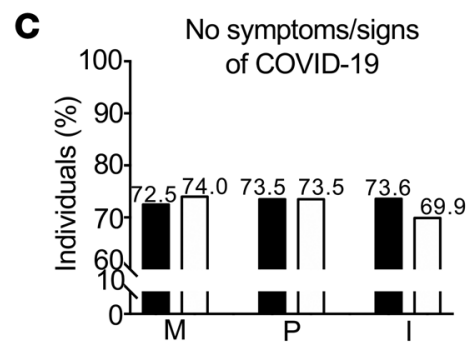

D

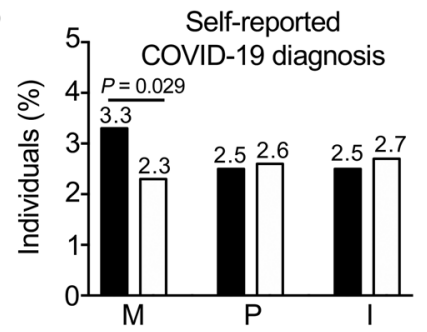

E

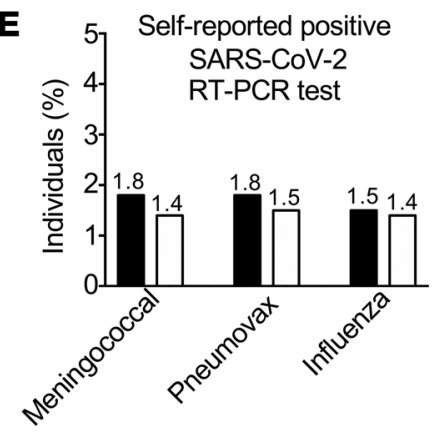

F Positive anti-SARS-CoV-2

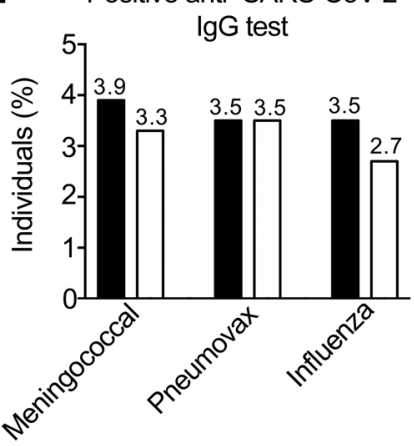

G

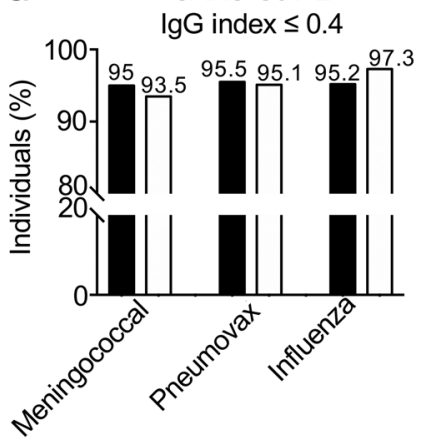

SARS-CoV-2

IgG index $>0.4$

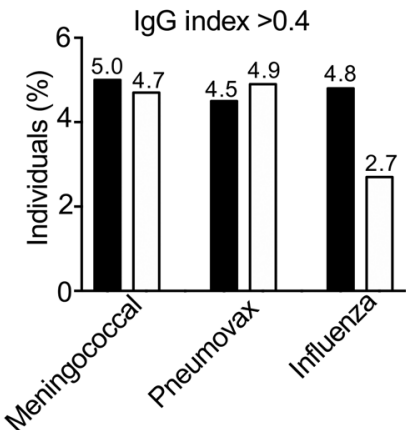

Figure 3. Meningococcal, Pneumovax, and influenza vaccinations are not associated with a beneficial effect of reducing COVID-19-related symptoms or SARS-CoV-2 infection. (A) Percentage of individuals in the entire cohort who reported a history of meningococcal $(n=1842)$, Pneumovax $(n=1007)$, or influenza $(n=6014)$ vaccination. (B) Percentage of individuals with a history of meningococcal, Pneumovax, or influenza vaccination who experienced COVID-19-related symptoms. (C) Percentage of individuals with or without a history of meningococcal, Pneumovax, or influenza vaccination who did not experience COVID-19-related symptoms during the prior 6 months. (D) Percentage of participants with or without a history of meningococcal, Pneumovax, or influenza vaccination who self-reported a medical diagnosis of COVID-19. (E) Percentage of participants with or without a history of meningococcal, Pneumovax, or influenza vaccination who self-reported a positive SARS-CoV-2 RT-PCR test. (F) Percentage of participants with or without a history of meningococcal, Pneumovax, or influenza vaccination with a positive anti-SARS-CoV-2 IgG test result. (C) Percentage of individuals with or without a history of meningococcal, Pneumovax, or influenza vaccination with a SARS-CoV-2 IgC index threshold of 0.4 or lower (left panel) or higher than 0.4. Significant $P$ values $(P<0.05)$ by $\chi^{2}$ test are displayed for each plot. 


\begin{tabular}{|c|c|c|}
\hline & \multicolumn{2}{|c|}{$\lg G$ index value $>0.4$} \\
\hline & OR (95\% Cl) & $P$ value \\
\hline \multicolumn{3}{|c|}{ Model: age- and sex-adjusted } \\
\hline BCG & $0.76(0.57,0.99)$ & 0.048 \\
\hline Meningococcal & $0.90(0.69,1.17)$ & 0.44 \\
\hline Pneumovax & $0.99(0.71,1.36)$ & 0.96 \\
\hline Influenza & $1.84(0.57,11.27)$ & 0.40 \\
\hline
\end{tabular}

exhibit characteristics of "trained" innate immune cells, such as heightened cytokine production, increased cellular metabolism, and epigenetic changes (57). It has been postulated that in some individuals, trained immunity may contribute to the initiation, maintenance, or aggravation of autoimmune and autoinflammatory disorders (57). In up to $20 \%$ of affected individuals, COVID-19 manifests as a severe disease characterized by a hyperinflammatory response and excessive production of proinflammatory cytokines (cytokine storm) (58). Therefore, it is possible that in promoting trained immunity, BCG vaccination could be deleterious by further fostering this hyperinflammatory response. However, a recent study of a large cohort of healthy volunteers demonstrated that BCG vaccination enhanced antimicrobial responses upon subsequent reinfection and simultaneously inhibited systemic inflammation in a sex-dependent manner (59). Moreover, a recent retrospective cohort study by Moorlag et al. demonstrated that BCG vaccination in the previous 5 years was safe and, similar to the findings presented here, associated with a decreased incidence of sickness during the COVID-19 pandemic (60). These findings are of high interest in the context of SARS-CoV-2 infection, as BCG vaccination might be protective by boosting the immune response against the virus and decreasing infection rates. BCG vaccination might also be beneficial in dampening the systemic inflammatory immune response in patients experiencing a severe hyperinflammatory course of COVID-19 (61).

Overall, in our cohort of HCWs, we observed a protective and nonspecific association between a history of BCG vaccination and reduced SARS-CoV-2 infection, seroprevalence, as well as presence of COVID-19-related symptoms. Our observation that BCG vaccination might be effective against SARS-CoV-2 infection and that it could potentially be used to prevent COVID-19 need to be further confirmed by the randomized, placebo-controlled, blinded clinical trials that are currently underway.

\section{Methods}

Study population. To determine whether a history of BCG vaccination is associated with decreased rates of SARS-CoV-2 infection, we analyzed data collected from a large cohort of HCWs who had undergone SARS-CoV-2 serologic testing. From May 11, 2020 to June 28, 2020, we enrolled 6679 active HCWs employed by the Cedars-Sinai Health System at multiple sites around Los Angeles County, including 2 major hospitals (Cedars-Sinai Medical Center and Marina Del Rey
Hospital) and several outpatient clinics affiliated with the Cedars-Sinai Medical Network (21). The study design and rationale were previously described (21). Among the 6679 enrolled participants, 6201 provided a blood sample used for SARS-CoV-2 IgG antibody testing and completed an electronic questionnaire regarding their vaccination history (BCG, influenza, meningococcal, and Pneumovax), as well as questions related to whether they had experienced COVID-19 symptoms in the 6 months prior to the blood sample collection. Participants also self-reported a previous COVID-19 diagnosis determined either by a MD or a positive SARS-CoV-2 RT-PCR test. If the participants took a SARS-CoV-2 RT-PCR test in the 6 months prior the study, they also self-reported whether the test was positive or negative. Race and ethnicity data were also collected by self-report. The following COVID-19-related symptoms were selected: fever, chills, dry cough, shortness of breath, muscle aches, fatigue, and reduced sense of smell or taste $(24,25)$.

Serologic assays. Quantification of SARS-CoV-2 IgG antibodies in the samples was performed at the Cedars-Sinai Department of Pathology and Laboratory Medicine using the Abbott Laboratories Architect SARS-CoV-2 IgG chemiluminescence microparticle immunoassay, according to the manufacturer's instructions. The signal-to-cutoff ratio (SC/CO) reported by the Abbott Architect SARS-CoV-2 IgG immunoassay corresponded to the RLU produced by the sample compared with the RLU produced by a test calibrator. The SC/CO recommended by the manufacturer to assign seropositivity status is 1.4 and has previously been validated for specificity and sensitivity up to 14 days after symptom onset (23). In addition, given prior reports (23) and our own laboratory findings that a cutoff point of 0.4 or higher was more sensitive for defining seropositivity and significantly associated with a prior COVID-19 diagnosis and symptoms, we also used the cutoff point of 0.4 or higher in our analysis. Notably, our main results were consistent for analyses using either the more stringent 1.4 or higher threshold or the more sensitive 0.4 or higher threshold.

Statistics. For analysis of the total study cohort, we used parametric tests to compare continuous and categorical variables. We also used bar charts to display COVID-related symptom frequency and seroprevalence status for the total cohort and then to show BCG, meningococcal, Pneumovax, and influenza vaccination status. We used logistic regression to examine the associations between vaccination status and SARS-CoV-2 IgG antibody status, adjusted for age and sex. All analyses were performed using R, version 4.0.1 (R Foundation for Statistical Computing). For all models, a 2-sided $P$ value at a threshold of 0.05 was considered statistically significant. $P$ values were determined by $t$ test or $\chi^{2}$ test and are shown in the figures.

Study approval. All study protocols were approved by the Cedars-Sinai Institutional Review Board, and all participants provided written informed consent before the blood sample and electronic survey form were collected.

\section{Author contributions}

JEE, JB, KS, JEVE, and SC conceived and designed the overall CORALE study. JEE, JB, KS, JEVE, and SC acquired the CORALE data. MA and MNR conceived the BCG and seroconversion analysis of the study, and JEE, MW, NS, JB, KS, JEVE, SC, and MA analyzed the data. MNR, SC, and MA drafted the manuscript, and all authors edited the manuscript. 


\section{Acknowledgments}

We thank the frontline HCWs at Cedars-Sinai who participated in this study for their dedication and high-quality care patient care. This work was supported by the National Cancer Institute, NIH (U54 CA26059) and the Erika J. Glazer Family Foundation.
Address correspondence to: Moshe Arditi, Cedars-Sinai Medical Center, 8700 Beverly Blvd., Davis Building, Rooms D4024, 4035, 4027, Los Angeles, CA 90048. Phone: 310.423.4461; Email: moshe.arditi@cshs.org.
1. WHO. Coronavirus Disease (COVID-19) Dashboard. https://covid19.who.int. Updated November 24, 2020. Accessed October 5, 2020.

2. Wu Z, McGoogan JM. Characteristics of and important lessons from the coronavirus disease 2019 (COVID-19) outbreak in china: summary of a report of 72314 cases from the chinese center for disease control and prevention. JAMA. 2020;323(13):1239-1242.

3. Huang $\mathrm{C}$, et al. Clinical features of patients infected with 2019 novel coronavirus in Wuhan, China. Lancet. 2020;395(10223):497-506.

4. Chen N, et al. Epidemiological and clinical characteristics of 99 cases of 2019 novel coronavirus pneumonia in Wuhan, China: a descriptive study. Lancet. 2020;395(10223):507-513.

5. Wang C, et al. A novel coronavirus outbreak of global health concern. Lancet. 2020;395(10223):470-473.

6. Benn CS, Netea MG, Selin LK, Aaby P. A small jab - a big effect: nonspecific immunomodulation by vaccines. Trends Immunol. 2013;34(9):431-439.

7. Biering-Sørensen S, et al. Early BCG-Denmark and neonatal mortality among infants weighing $<2500$ g: a randomized controlled trial. Clin Infect Dis. 2017;65(7):1183-1190.

8. Garly ML, et al. BCG scar and positive tuberculin reaction associated with reduced child mortality in West Africa. A non-specific beneficial effect of BCG? Vaccine. 2003;21(21-22):2782-2790.

9. Biering-Sørensen S, et al. Small randomized trial among low-birth-weight children receiving bacillus Calmette-Guérin vaccination at first health center contact. Pediatr Infect Dis J. 2012;31(3):306-308.

10. Kristensen I, et al. Routine vaccinations and child survival: follow up study in Guinea-Bissau, West Africa. BMJ. 2000;321(7274):1435-1438.

11. Hirve S, et al. Non-specific and sex-differential effects of vaccinations on child survival in rural western India. Vaccine. 2012;30(50):7300-7308.

12. Aaby P, et al. Randomized trial of BCG vaccination at birth to low-birth-weight children: beneficial nonspecific effects in the neonatal period? J Infect Dis. 2011;204(2):245-252.

13. Nemes E, et al. Prevention of M. tuberculosis infection with H4:IC31 vaccine or BCG revaccination. N EnglJMed. 2018;379(2):138-149.

14. Wardhana null, et al. The efficacy of bacillus Calmette-Guérin vaccinations for the prevention of acute upper respiratory tract infection in the elderly. Acta Med Indones. 2011;43(3):185-190.

15. Glasgow LA, et al. Immunomodulation of host resistance to experimental viral infections in mice: effects of Corynebacterium acnes, Corynebacterium parvum, and bacille Calmette-guérin. Infect Dis. 1977;135(5):763-770.

16. Blok BA, et al. Trained innate immunity as underlying mechanism for the long-term, nonspecific effects of vaccines. J Leukoc Biol.
2015;98(3):347-356.

17. Netea MG, et al. Trained immunity: A program of innate immune memory in health and disease. Science. 2016;352(6284):aaf1098.

18. Mangtani $\mathrm{P}$, et al. The duration of protection of school-aged BCG vaccination in England: a population-based case-control study. Int J Epidemiol. 2018;47(1):193-201.

19. Chumakov K, et al. Can existing live vaccines prevent COVID-19? Science. 2020;368(6496):1187-1188.

20. Fidel PL, Noverr MC. Could an unrelated live attenuated vaccine serve as a preventive measure to dampen septic inflammation associated with COVID-19 infection? mBio. 2020;11(3):e00907-20.

21. Ebinger JE, et al. An opportune and relevant design for studying the health trajectories of healthcare workers [preprint]. https://doi.org/10. 1101/2020.06.30.20140046. Posted on medRxiv July 3, 2020.

22. County of Los Angeles Public Health Department. LA County COVID-19 Surveillance Dashboard. http://dashboard.publichealth.lacounty.gov/ covid19_surveillance_dashboard. Updated November 11, 2020. Accessed September 182020.

23. Bryan A, et al. Performance characteristics of the Abbott Architect SARS-CoV-2 IgG assay and seroprevalence in Boise, Idaho. JClin Microbiol. 2020;58(8):e00941-20.

24. CDC. Symptoms of coronavirus. https://www. cdc.gov/coronavirus/2019-ncov/symptoms-testing/symptoms.html. Updated May 13, 2020. Accessed November 24, 2020.

25. Wise J. COVID-19: study reveals six clusters of symptoms that could be used as a clinical prediction tool. BMJ. 2020;370:m2911.

26. Guan WJ, et al. Comorbidity and its impact on 1590 patients with COVID-19 in China: a nationwide analysis. Eur Respir J. 2020;55(5):2000547.

27. Richardson S, et al. Presenting characteristics, comorbidities, and outcomes among 5700 patients hospitalized with COVID-19 in the New York City area. JAMA. 2020;323(20):2052-2059.

28. Stokes EK, et al. Coronavirus disease 2019 case surveillance-United States, January 22-May 30, 2020. MMWR Morb Mortal Wkly Rep. 2020;69(24):759-765

29. Giamarellos-Bourboulis EJ, et al. ACTIVATE: randomized clinical trial of $\mathrm{BCG}$ vaccination against infection in the elderly. Cell. 2020;183(2):315-323.e9.

30. O'Neill LAJ, Netea MG. BCG-induced trained immunity: can it offer protection against COVID19? Nat Rev Immunol. 2020;20(6):335-337.

31. Netea MG, et al. Trained immunity: a tool for reducing susceptibility to and the severity of SARSCoV-2 infection. Cell. 2020;181(5):969-977.

32. Kleen TO, et al. Mitigating coronavirus induced dysfunctional immunity for at-risk populations in COVID-19: trained immunity, BCG and "new old friends". Front Immunol. 2020;11:2059.

33. Berg MK, et al. Mandated bacillus CalmetteGuérin (BCG) vaccination predicts flattened curves for the spread of COVID-19. Sci Adv. 2020;6(32):eabc1463.

34. Escobar LE, Molina-Cruz A, Barillas-Mury C. BCG vaccine protection from severe coronavirus disease 2019 (COVID-19). Proc Natl Acad Sci US A. 2020;117(30):17720-17726

35. Moscola J, et al. Prevalence of SARS-CoV-2 antibodies in health care personnel in the New York City area. JAMA. 2020;324(9):893-895.

36. Sood N, et al. Seroprevalence of SARS-CoV-2specific antibodies among adults in Los Angeles County, California, on April 10-11, 2020. JAMA. 2020;323(23):2425-2427.

37. Jensen A, et al. Early childhood vaccination and subsequent mortality or morbidity: are observational studies hampered by residual confounding? A Danish register-based cohort study. BMJ Open. 2019;9(9):e029794.

38. Sørup S, et al. Oral polio vaccination and hospita admissions with non-polio infections in denmark: nationwide retrospective cohort study. Open Forum Infect Dis. 2016;3(1):ofv204.

39. Seppälä E, et al. Viral interference induced by live attenuated virus vaccine (OPV) can prevent otitis media. Vaccine. 2011;29(47):8615-8618.

40. Aronson NE, et al. Long-term efficacy of BCG vaccine in American Indians and Alaska Natives: A 60-year follow-up study. JAMA. 2004;291(17):2086-2091.

41. Usher NT, et al. Association of BCG vaccination in childhood with subsequent cancer diagnoses: a 60-year follow-up of a clinical trial. JAMA Netw Open. 2019;2(9):e1912014.

42. Katelaris AL, et al. Effectiveness of BCG vaccination against mycobacterium tuberculosis infection in adults: a cross-sectional analysis of a UK-based cohort. JInfect Dis. 2020;221(1):146-155.

43. Nguipdop-Djomo P, et al. Duration of BCG protection against tuberculosis and change in effectiveness with time since vaccination in Norway: a retrospective population-based cohort study. Lancet Infect Dis. 2016;16(2):219-226.

44. Kleinnijenhuis J, et al. Long-lasting effects of BCG vaccination on both heterologous Th1/Th17 responses and innate trained immunity. JInnate Immun. 2014;6(2):152-158.

45. Quintin J, et al. Candida albicans infection affords protection against reinfection via functional reprogramming of monocytes. Cell Host Microbe. 2012;12(2):223-232.

46. Arts RJW, et al. BCG vaccination protects against experimental viral infection in humans through the induction of cytokines associated with trained immunity. Cell Host Microbe. 2018;23(1):89-100.e5.

47. Kleinnijenhuis J, et al. BCG-induced trained immunity in NK cells: role for non-specif- 
ic protection to infection. Clin Immunol. 2014;155(2):213-219.

48. Mazzoni A, et al. Impaired immune cell cytotoxicity in severe COVID-19 is IL-6 dependent. J Clin Invest. 2020;130(9):4694-4703.

49. Giamarellos-Bourboulis EJ, et al. Complex immune dysregulation in COVID-19 patients with severe respiratory failure. Cell Host Microbe. 2020;27(6):992-1000.e3.

50. Braun J, et al. SARS-CoV-2-reactive T cells in healthy donors patients with COVID-19. Nature. 2020;587(7833):270-274.

51. Mateus J, et al. Selective cross-reactive SARSCoV-2 $\mathrm{T}$ cell epitopes in unexposed humans. Science. 2020;370(6512):89-94.

52. Grifoni A, et al. Targets of $\mathrm{T}$ cell responses to SARS-CoV-2 coronavirus in humans with
COVID-19 disease and unexposed individuals. Cell.2020;181(7):1489-1501.e15.

53. Le Bert N, et al. SARS-CoV-2-specific T cell immunity in cases of COVID-19 and SARS, and uninfected controls. Nature. 2020;584(7821):457-462.

54. Sette A, Crotty S. Pre-existing immunity to SARSCoV-2: the knowns and unknowns. Nat Rev Immunol. 2020;20(8):457-458.

55. Fjällbrant $\mathrm{H}$, et al. Primary vaccination and revaccination of young adults with BCG: a study using immunological markers. Scand J Infect Dis. 2007;39(9):792-798.

56. Tomita $Y$, et al. BCG vaccine may generate cross-reactive $\mathrm{T}$ cells against SARS-CoV-2: In silico analyses and a hypothesis. Vaccine. 2020;38(41):6352-6356.
57. Arts RJW, et al. The potential role of trained immunity in autoimmune and autoinflammatory disorders. Front Immunol. 2018;9:298.

58. Mehta P, et al. COVID-19: consider cytokine storm syndromes and immunosuppression. Lancet. 2020;395(10229):1033-1034.

59. Koeken VA, et al. BCG vaccination in humans inhibits systemic inflammation in a sex-dependent manner. JClin Invest. 2020;130(10):5591-5602.

60. Moorlag SJCFM, et al. Safety and COVID-19 symptoms in individuals recently vaccinated with BCG: a retrospective cohort study. Cell Rep Med. 2020;1(5):100073.

61. Prentice S, Dockrell HM. Antituberculosis BCG vaccination: more reasons for varying innate and adaptive immune responses. JClin Invest. 2020;130(10):5121-5123. 